\section{Persistence of SARS-CoV-2- Specific IgG in Children 6 Months After Infection, Australia}

\author{
Zheng Quan Toh, Rachel A. Higgins, Lien Anh Ha Do, \\ Karin Rautenbacher, Francesca L. Mordant, \\ Kanta Subbarao, Kate Dohle, Jill Nguyen, \\ Andrew C. Steer, Shidan Tosif, Nigel W. Crawford, \\ Kim Mulholland, Paul V. Licciardi
}

\begin{abstract}
Author affiliations: Murdoch Children's Research Institute, Melbourne, Victoria, Australia (Z.Q. Toh, R.A. Higgins, L.A.H. Do, K. Dohle, J. Nguyen, A.C. Steer, S. Tosif, N.W. Crawford, K. Mulholland, P.V. Licciardi); The University of Melbourne, Melbourne (Z.Q. Toh, L.A.H. Do, A.C. Steer, S. Tosif, N.W. Crawford, K. Mullholland, P.V. Licciardi); The Royal Children's Hospital, Melbourne (K. Rautenbacher, A.C. Steer, S. Tosif, N.W. Crawford); World Health Organization Collaborating Centre for Reference and Research on Influenza, The Peter Doherty Institute for Infection and Immunity, Melbourne (F.L. Mordant, K. Subbarao); London School of Hygiene and Tropical Medicine, London, UK (K. Mulholland)
\end{abstract}

\section{DOI: https://doi.org/10.3201/eid2708.210965}

The duration of the humoral immune response in children infected with severe acute respiratory syndrome coronavirus 2 is unknown. We detected specific IgG 6 months after infection in children who were asymptomatic or had mild symptoms of coronavirus disease. These findings will inform vaccination strategies and other prevention measures.

C hildren $<18$ years of age account for $\approx 3 \%$ of coronavirus disease (COVID-19) cases worldwide (1). Most $(70 \%)$ children with COVID-19 are asymptomatic or have mild illness; very few require hospitalization $(2,3)$. The nature and persistence of the immune response generated by children after infection with severe acute respiratory syndrome coronavirus 2 (SARS-CoV-2), the causative agent of COVID-19, is unknown. We investigated the humoral immune response to SARS-CoV-2 in children and adults as part of a longitudinal cohort study in Melbourne, Victoria, Australia.

Nasopharyngeal swab samples of persons with suspected SARS-CoV-2 infection and their close contacts were tested by reverse transcription PCR at The Royal Children's Hospital in Melbourne during MayOctober 2020. We invited SARS-CoV-2-positive patients and their household members to participate in this cohort study. We collected blood samples at the time of enrollment, as well as $\approx 28$ days, 3 months, and 6 months later. We obtained written informed consent from parents/guardians and assent from children. The study was conducted with the approval of the Human Research Ethics Committee at The Royal Children's Hospital (approval no. HREC/63666/ RCHM-2019).

To measure IgG, we used a modified 2-step ELISA based on the method described by Amanat et al. (4) and the LIAISON SARS-CoV-2 S1/S2 IgG assay (DiaSorin, https://www.diasorin.com). We also conducted a SARS-CoV-2 microneutralization assay on an available subset of samples. For the ELISA, we screened samples using the SARS-CoV-2 receptorbinding domain as the antigen; for potential positive samples, we confirmed results that tested positive by additional ELISA using S1 antigen. We calculated the results of S1-positive samples according to the World Health Organization SARS-CoV-2 pooled serum standard (standard provided by the National Institute for Biological Standards and Control, South Mimms, UK) and reported data as ELISA units per milliliter. We set a seropositivity cutoff at 1.5 ELISA units $/ \mathrm{mL}$ on the basis of results of archived serum samples taken before the pandemic. We then conducted the LIAISON assay according to the manufacturer's instructions and the microneutralization assay as described by Tosif et al. (5) (Appendix, https://wwwnc.cdc. gov/EID/article/27/8/21-0965-App1.pdf).

During May 10, 2020-October 28, 2020, we recruited a cohort of 134 children ( $0-18$ years of age) and 160 adults (19-73 years of age). We included only participants with a positive PCR result for SARS$\mathrm{CoV}-2$ or who were seropositive at the first timepoint (median 11 days after diagnosis, range 5-13 days) and had blood samples for $\geq 2$ timepoints. At the first timepoint, 4 adults had negative PCR results but positive serologic results; of these adults, 3 had borderline seropositive antibody levels.

By February 2021, we had identified 54 SARSCoV-2-positive participants: 22 children (median age of 4 years, range $0-18$ years) and 32 adults (median age of 37 years, range 22-73 years). In total, 5 (23\%) children and $2(6 \%)$ adults were asymptomatic; the rest had mild symptoms, and none were hospitalized. The median duration of follow-up after diagnosis was 195 days (range 188-213 days) for children and 194 days (range 183-212 days) for adults.

By day 43 (range 27-79), 15/19 (79\%) children and 26/28 (93\%) adults had seroconverted. These participants remained seropositive for $\geq 90$ days (Figure, panels A, B). By day 195 ( $\approx 6$ months), 14/17 (82\%) of children and 18/21 (86\%) of adults were seropositive; however, from day 43 to 195, geometric mean antibody 


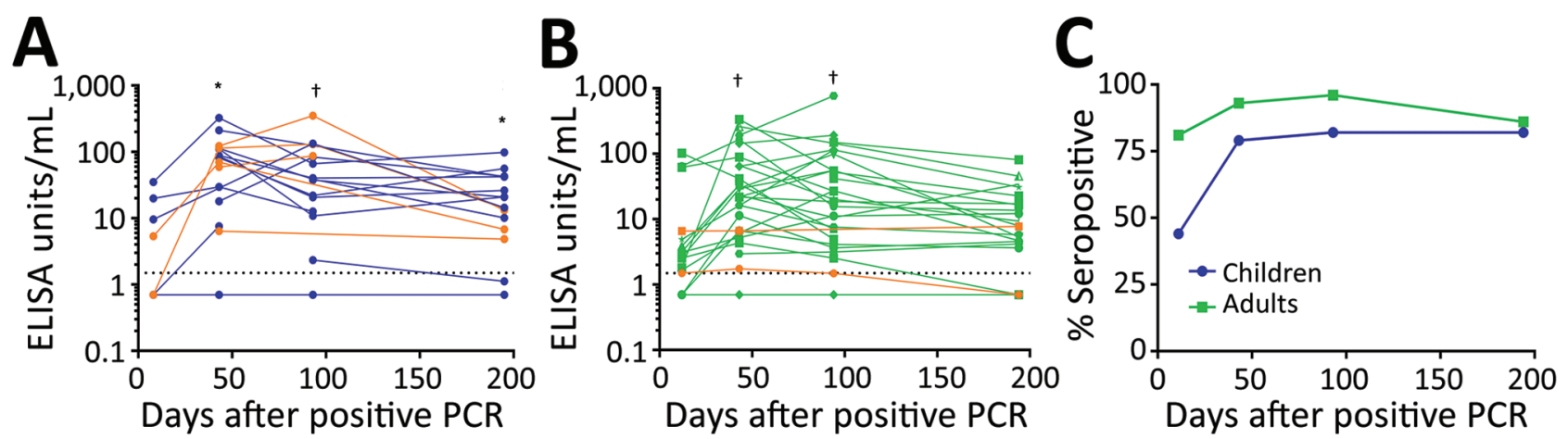

Figure. Persistence of $\lg G$ responses against severe acute respiratory syndrome coronavirus 2 in children and adults, Australia, 2020-2021. Patients tested positive by PCR, ELISA, or both. A) Antibody responses of 22 children 0-18 years of age. B) Antibody responses of 32 adults 22-73 years of age. Orange points and lines indicate asymptomatic cases; blue points and lines indicate symptomatic cases in children; green points and lines indicate symptomatic cases in adults. Dotted lines indicate seropositivity cutoff. C) Seropositivity rates in 22 children and 32 adults. Blue points and lines indicate all children, regardless of symptoms; green points and lines indicate all adults, regardless of symptoms. ${ }^{*} p<0.05 ; \uparrow p<0.01$ (compared with the fist timepoint [day 11]).

concentration decreased $\approx 2$-fold in both groups (Figure, panel C). We observed no significant differences in geometric mean antibody concentration from day 43 (range 27-79) to day 194 (range 183-212), nor from 93 (range 27-79) to day 194 (range 183-212), for either children or adults (Figure, panels A, B). The seropositivity and antibody levels were also not significantly different between children and adults at all timepoints (Figure 1, panel C; Appendix Figure 1). Seropositive samples defined by our in-house ELISA correlated with results from the LIAISON assay and neutralizing antibody assay (Appendix Figures 2, 3). In total, 4/19 (21\%) children and 2/28 (7\%) adults did not seroconvert; however, we could not rule out other SARS-CoV-2-related immune responses, such as cellular or mucosal mechanisms $(5,6)$.

We found that, similar to the adults in this cohort and those in previous studies $(7,8)$, SARS-CoV-2-positive children with no or mild symptoms mounted strong and durable humoral responses that persisted for $\geq 6$ months. Our study was limited by the relatively small sample size; in addition, only a subset of samples was available for the microneutralization assay. In conclusion, our data indicate that SARS-CoV-2-positive children have a persistent antibody response for $\geq 6$ months. The roles and durations of other components of the immune system (such as the cellular and mucosal responses) during SARS-CoV-2 infection remain undetermined. These results will inform vaccination strategies and other public health measures.

\section{Acknowledgments}

We thank the study participants and families for their involvement. We also acknowledge the Murdoch Children's Research Institute Biobanking Facility for their help in processing the samples.
Funding for the recruitment of participants was provided by the Royal Children's Hospital Foundation (grant no. 2020-1293) and the Infection and Immunity Theme, Murdoch Children's Research Institute. P.V.L. is supported by a National Health and Medical Research Council Career Development Fellowship. K.S. is supported by a National Health and Medical Research Council Investigator grant; her research is supported by funding from the Jack Ma Foundation and the a2 Milk Company. N.W.C. received funding from Centers of Excellence in Influenza Research and Surveillance-CrossCenter Southern Hemisphere Project, National Institute of Health (grant no. HHSN272201400005, subaward no. 417760-G/GR511065). This work is financially and logistically supported by Victorian Government's Medical Research Operational Infrastructure Support Program. The Melbourne WHO Collaborating Centre for Reference and Research on Influenza is financially and logistically supported by the Australian Government Department of Health.

\section{About the Author}

Dr. Toh is a research officer based at the Murdoch Children's Research Institute, Melbourne, Victoria, Australia. His primary research interests include infectious diseases, immunology, and vaccinology.

\section{References}

1. Dawood FS, Ricks P, Njie GJ, Daugherty M, Davis W, Fuller JA, et al. Observations of the global epidemiology of COVID-19 from the prepandemic period using web-based surveillance: a cross-sectional analysis. Lancet Infect Dis. 2020;20:1255-62. https:/ / doi.org/10.1016/ S1473-3099(20)30581-8

2. Qiu H, Wu J, Hong L, Luo Y, Song Q, Chen D. Clinical and epidemiological features of 36 children with coronavirus 
disease 2019 (COVID-19) in Zhejiang, China: an observational cohort study. Lancet Infect Dis. 2020;20:689-96. https://doi.org/10.1016/S1473-3099(20)30198-5

3. Zimmermann P, Curtis N. Why is COVID-19 less severe in children? A review of the proposed mechanisms underlying the age-related difference in severity of SARS-CoV-2 infections. Arch Dis Child. 2020 Dec 1 [Epub ahead of print].

4. Amanat F, Stadlbauer D, Strohmeier S, Nguyen THO, Chromikova V, McMahon M, et al. A serological assay to detect SARS-CoV-2 seroconversion in humans. Nat Med. 2020;26:1033-6. https:/ / doi.org/10.1038/s41591-020-0913-5

5. Tosif S, Neeland MR, Sutton P, Licciardi PV, Sarkar S, Selva KJ, et al. Immune responses to SARS-CoV-2 in three children of parents with symptomatic COVID-19. Nat Commun. 2020;11:5703. https:// doi.org/10.1038/ s41467-020-19545-8

6. Sekine T, Perez-Potti A, Rivera-Ballesteros O, Strålin K, Gorin JB, Olsson A, et al.; Karolinska COVID-19 Study Group. Robust T cell immunity in convalescent individuals with asymptomatic or mild COVID-19. Cell. 2020;183:158-168.e14. https:/ / doi.org/10.1016/ j.cell.2020.08.017

7. Duysburgh E, Mortgat L, Barbezange C, Dierick K, Fischer N, Heyndrickx L, et al. Persistence of IgG response to SARS-CoV-2 [Erratum in: Lancet Infect Dis. 2021;21:e16; 2021;21:e36]. Lancet Infect Dis. 2021;21;163-164.

8. Choe PG, Kim KH, Kang CK, Suh HJ, Kang E, Lee SY, et al. Antibody responses 8 months after asymptomatic or mild SARS-CoV-2 infection. Emerg Infect Dis. 2021;27:928-31. https://doi.org/10.3201/eid2703.204543

Address for correspondence: Paul V. Licciardi, Murdoch Children's Research Institute, Royal Children's Hospital, Flemington Road, Parkville, VIC 3052, Australia; email: paul. licciardi@mcri.edu.au

\section{COVID-19 and the Consequences of Anchoring Bias}

\author{
Harold W. Horowitz, Caren Behar, Jeffrey Greene \\ Author affiliations: Weill Cornell Medicine, New York, New York, \\ USA (H.W. Horowitz); New York-Presbyterian Brooklyn \\ Methodist Hospital, Brooklyn, New York, USA (H.W. Horowitz); \\ New York University Langone School of Medicine, New York \\ (C. Behar, J. Greene)
}

DOI: https://doi.org/10.3201/eid2708.211107
Suspicion of coronavirus disease in febrile patients might lead to anchoring bias, causing misdiagnosis of other infections for which epidemiologic risks are present. This bias has potentially severe consequences, illustrated by cases of human granulocytic anaplasmosis and Lyme disease in a pregnant woman and human granulocytic anaplasmosis in another person.

Coronavirus disease (COVID-19) took the United States by force during the first quarter of 2020, affecting the economy, societal norms, and the delivery of medical care $(1,2)$. As fear of COVID-19 has spread, diagnosing COVID-19 in febrile persons has been prioritized, and patients may be presumed to have COVID-19 pending results of testing for severe acute respiratory syndrome coronavirus 2 (SARS-CoV-2). This mindset has had unintended consequences, including delaying evaluations for other infectious diseases, potentially leading to adverse outcomes. We describe 2 cases that illustrate this point.

In the first case, a 35-year-old man left New York, New York, USA, to go hiking in Maryland during June 5-June 7, 2020. He experienced fever, body aches, and fatigue during June 10-13 that resolved but left him fatigued and weak. He was seen on June 19; laboratory results were unremarkable, but lymphopenia was detected. He tested negative for SARS-CoV-2 on June 19 and June 25 by PCR. On June 25, ELISA for Lyme disease was positive, and reflex to Western blot revealed IgM 41-kD, 39-kD, and 23-kD bands but no IgG bands. Fever up to $38^{\circ} \mathrm{C}$ recurred on June 22 and lasted until June 29; he also experienced persistent fatigue and myalgia. Further testing on July 6 revealed serologic results for Lyme similar to results from June 25 and Anaplasma phagocytophilum titers of IgM 1:320 and IgG 1:1260. Anaplasma PCR was negative on that date. He was treated with doxycycline for 10 days and recovered.

In the second case, a 31-year-old woman who was 6 months pregnant left New York at the end of May 2020 to rent a house in Ulster County, New York. On June 3, she removed a tick from her neck. On June 9, she experienced severe headaches and the next day had low-grade fever, chills, and body aches. She had no cough, shortness of breath, or sore throat. On June 10 , she tested negative for SARS-CoV-2 by PCR. She continued to have extreme fatigue, myalgia, and lowgrade fever. She was prescribed oseltamivir by her obstetrician on June 11. On June 14, she felt better. Repeat PCR testing for SARS-CoV-2 on June 15 was negative. She continued to improve until June 23 , when she experienced recurrent fever up to $38.9^{\circ} \mathrm{C}$, chills, and lethargy. She contacted her obstetrician and was told she had 